\title{
Angiopoietins have distinct modular domains essential for receptor binding, dimerization and superclustering
}

Samuel Davis, Nick Papadopoulos, Thomas H. Aldrich, Peter C. Maisonpierre, Tammy Huang, Lubomir Kovac, April Xu, Raymond Leidich, Elzbieta Radziejewska, Ashique Rafique, Judah Goldberg, Vivek Jain, Kevin Bailey, Margaret Karow, Jim Fandl, Steven J. Samuelsson, Ella loffe, John S. Rudge, Thomas J. Daly, Czeslaw Radziejewski and George D. Yancopoulos

Nat. Struct. Biol. 10, 38-44 (2003).

Fig. 5 of this paper contains a mistake. The order of panels $(b)$ and $(c)$ should be reversed. For clarity, the entire figure is reprinted. We apologize for any inconvenience this may have caused.

$a$

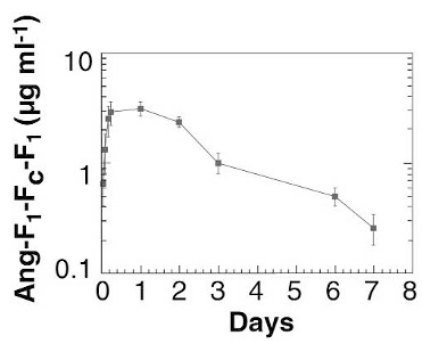

$b$

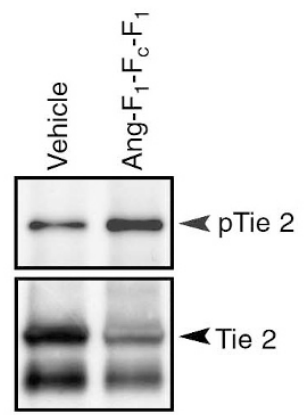

C

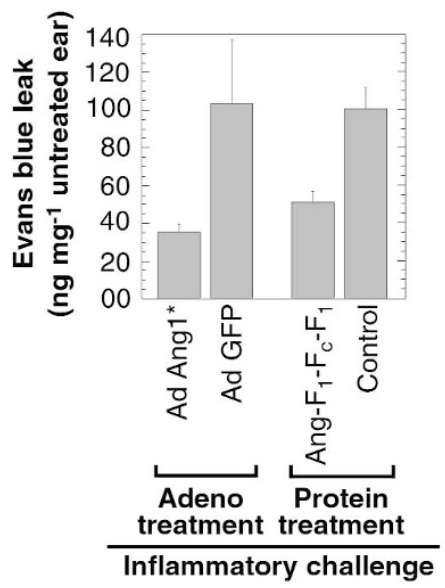

Fig. 5 Engineered tetramers of Ang1 are useful in vivo reagents. $a$, Pharmacokinetic analysis of Ang- $F_{1}-F_{c}-F_{1}$ in mice, depicting circulating levels at indicated times after subcutaneous injection. $\boldsymbol{b}$, In vivo phosphorylation of Tie2 stimulated by subcutaneous injection of Ang- $F_{1}-F_{c}-F_{1}$, assayed $24 h$ after injection. Tie2 was immunoprecipitated from $1 \mathrm{mg}$ of lung lysate and immunoblotted either with anti-phosphotyrosine to detect phosphory-

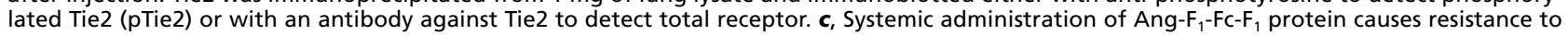
mustard oil-induced vascular leakage comparable to resistance caused by adenoviral gene delivery. Plasma leakage of Evans blue was measured as described ${ }^{19}$ in mice treated with Ang $-\mathrm{F}_{1}-\mathrm{Fc}-\mathrm{F}_{1}$ protein or with adenoviral vectors expressing either Ang 1 * (Ad Ang $\left.1 *\right)$ or green fluorescent protein (Ad GFP) as a control. Data are expressed as mean \pm SEM (standard error of the mean) for 3 ears per group ( $n=3$ ).

\section{erratum}

\section{Moving across membranes}

\author{
Elaine A. Neale
}

Nat. Struct. Biol. 10, 2-3 (2003).

Reference 6 was inadvertently omitted from the reference list during the production process of this report. The missing reference is printed below. We apologize for any inconvenience this may have caused.

6. Ren, J. et al. Science 284, 955-957 (1999). 